\title{
Auroral hiss, electron beams and standing Alfvén wave currents near Saturn's moon Enceladus
}

\author{
D. A. Gurnett, ${ }^{1}$ T. F. Averkamp, ${ }^{1}$ P. Schippers, ${ }^{1}$ A. M. Persoon, ${ }^{1}$ G. B. Hospodarsky, ${ }^{1}$ \\ J. S. Leisner, ${ }^{1}$ W. S. Kurth, ${ }^{1}$ G. H. Jones, ${ }^{2}$ A. J. Coates, ${ }^{2}$ F. J. Crary, ${ }^{3}$ \\ and M. K. Dougherty ${ }^{4}$
}

Received 21 January 2011; revised 16 February 2011; accepted 23 February 2011; published 24 March 2011.

[1] Observations from the Cassini spacecraft have shown that Saturn's small icy moon Enceladus ejects a plume of water vapor and small ice particles into Saturn's rapidly co-rotating magnetosphere. In this paper we show that the interaction of the moon with the magnetospheric plasma produces a number of electrodynamics effects that are remarkably similar to those observed in Earth's auroral regions and near Jupiter's moon Io. These include whistler-mode emissions similar to terrestrial auroral hiss, magnetic-field-aligned electron beams, and currents associated with a standing Alfvén wave excited by the moon. Ray path analyses of the auroral hiss show that the electron beams responsible for the emissions are accelerated very close to the moon, most likely by parallel electric fields associated with the Alfvén wave. However, other possibilities such as electric fields due to electrostatic charging of the moon's surface or of particles in the water vapor plume should be considered. Citation: Gurnett, D. A., et al. (2011), Auroral hiss, electron beams and standing Alfvén wave currents near Saturn's moon Enceladus, Geophys. Res. Lett., 38, L06102, doi:10.1029/2011GL046854.

\section{Introduction}

[2] Starting in early 2005 the Cassini spacecraft, which is in orbit around Saturn, began a series of close flybys of Saturn's moon Enceladus. This small icy moon has a radius of only $252 \mathrm{~km}$ and orbits Saturn at a radial distance of 3.95 $\mathrm{R}_{\mathrm{S}}$ (Radius of Saturn $=60,268 \mathrm{~km}$ ). From previous Voyager observations it was known that the moon displays evidence of geological activity, and was also thought to be the origin of small micron-sized particles in the E ring [Smith et al., 1981]. During the first Cassini flyby of Enceladus on 17 February 2005, magnetic field perturbations were observed in the vicinity of the moon that were attributed to the interaction of Saturn's rapidly co-rotating magnetospheric plasma with a dense cloud of gas near the moon [Dougherty et al., 2006]. Subsequent flybys confirmed the existence of the gas cloud and showed that the cloud originated from a plume of water vapor and small particles escaping from geyser-like vents near the south pole of the moon [Porco

\footnotetext{
${ }^{1}$ Department of Physics and Astronomy, University of Iowa, Iowa City, Iowa, USA.

${ }^{2}$ Mullard Space Science Laboratory, University College London, Dorking, UK.

${ }^{3}$ Southwest Research Institute, San Antonio, Texas, USA.

${ }^{4}$ Blackett Laboratory, Imperial College London, London, UK.
}

Copyright 2011 by the American Geophysical Union. 0094-8276/11/2011GL046854 et al., 2006]. Plasma measurements also showed that the water vapor cloud undergoes a strong charge-exchange interaction with the co-rotating plasma, with some of the pickup ions being accelerated to as much as a hundred $\mathrm{eV}$ by the co-rotational electric field [Tokar et al., 2006]. More recently, Pryor et al. [2011] reported observations of aurora near the foot of the magnetic flux tube passing through the moon, indicative of a strong electrodynamics coupling to Saturn's atmosphere. In this paper we report the discovery of whistler-mode auroral hiss emissions produced by magnetic field-aligned electron beams accelerated near the moon, most likely by parallel electric fields associated with a standing Alfvén wave (sometimes called an Alfvén wing) excited by the moon.

\section{Auroral Hiss}

[3] Auroral hiss is a broadband whistler-mode emission that is commonly observed in Earth's auroral regions. First shown to be associated with the aurora by ground-based radio observations, auroral hiss has been extensively studied by both low and high altitude Earth-orbiting spacecraft [Gurnett, 1966; Gurnett et al., 1983; Ergun et al., 2003]. The basic identifying feature of auroral hiss is a characteristic V-shaped frequency-time spectrum, also sometimes called a "saucer" or a "funnel." The V-shaped spectrum is a propagation effect that arises for quasi-electrostatic whistlermode waves propagating at wave normal angles near the resonance cone [Mosier and Gurnett, 1969]. Comparisons with charged particle measurements have shown that auroral hiss is generated by magnetic field-aligned electron beams with energies ranging from a few tens of eV to several $\mathrm{keV}$ [Gurnett and Frank, 1972; Ergun et al., 2003]. Although once thought to be produced by incoherent Cerenkov radiation, auroral hiss is now believed to be generated by a coherent beam-plasma instability [Maggs, 1976] at the Landau resonance velocity, $\mathrm{v}_{\|=\omega} / \mathrm{k}_{\|}$.

\section{Observations Near Enceladus}

[4] At the time of this writing (31 December 2010) a total of fourteen flybys of Enceladus have been carried out by Cassini. A summary of these flybys is given by Table $\mathrm{S} 1$ of the auxiliary material. ${ }^{1}$ Upon examining the data from the Cassini Radio and Plasma Wave Science (RPWS) instrument we discovered that six had V-shaped auroral hiss emissions. Of these, only three had suitable pitch angle coverage from the Cassini Plasma Spectrometer (CAPS) to

\footnotetext{
${ }^{1}$ Auxiliary materials are available in the HTML. doi:10.1029/ 2011 GL046854.
} 


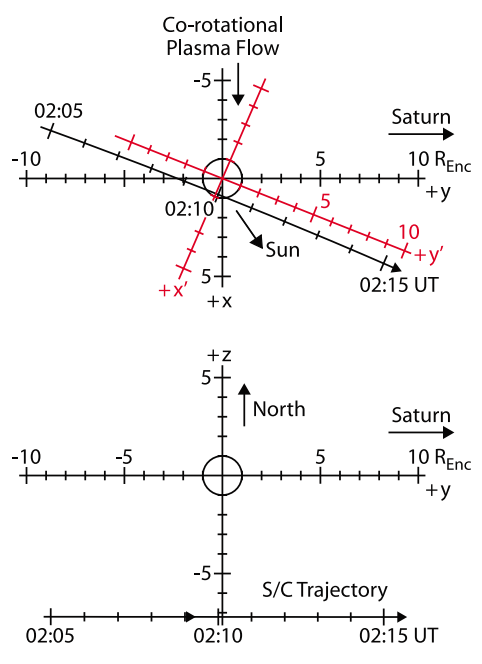

E8, Enceladus Flyby, Nov. 21, Day 325, 2009

Figure 1. (top and bottom) The spacecraft trajectory in an Enceladus-centered co-rotationally aligned coordinate system. The $\mathrm{z}$ axis is parallel to Saturn's rotational axis, the $+x$ axis is in the direction of the nominal co-rotational plasma flow, and the $+y$ axis is directed toward Saturn. The rotated, $\left(x^{\prime}, y^{\prime}\right)$, coordinate system (red), with the $y^{\prime}$ axis parallel to the spacecraft trajectory, is used in the ray path analysis (see Figure 3).

search for simultaneous field-aligned electron beams. In this paper we describe a particularly clear example that occurred during the E8 flyby on 21 November 2009. The spacecraft trajectory relative to Enceladus during this flyby is shown in Figure 1. As can be seen, the spacecraft passed almost directly south of the moon at a radial distance of about $7.2 \mathrm{R}_{\text {Enc }}$ while moving toward Saturn roughly perpendicular to the co-rotational plasma flow. An electric field frequency-time spectrogram of the plasma wave emissions observed during the flyby is shown in Figure 2a. The spectrogram shows that a very clear V-shaped auroral hiss emission occurred as the spacecraft passed over the south pole of the moon. The emission is centered almost exactly at the time, 02:10:13 UT (Universal Time), that the spacecraft passed closest to the magnetic field line through the center of the moon. This time is shown by the arrow at the top of the spectrogram, and the projected size of the magnetic flux tube through Enceladus is indicated by the hatched region.

[5] By good fortune during this flyby the field of view of the CAPS electron spectrometer (ELS) was aligned in the proper direction to detect electrons arriving from the moon, and two well-defined electron beams were observed. Figure $2 \mathrm{~b}$ shows an energy-time spectrogram of these beams, and Figure 2c shows the corresponding pitch angles. The first beam, which occurred from about 02:09:30 to 02:09:45 UT, has energies ranging from about 1 to $50 \mathrm{eV}$, and the second beam, which occurred from about 02:10:05 to $02: 10: 40$ UT has energies ranging from about 1 to $10 \mathrm{eV}$. In both cases the pitch angles are less than $20^{\circ}$, indicating that the beams are very nearly aligned with the magnetic field. It can be easily verified that these electron energies are in the proper range to be in Landau resonance with short wavelength whistler-mode waves propagating along the resonance cone (i.e., the auroral hiss).

[6] Since electron beams are often associated with fieldaligned currents, we also examined the Cassini magnetometer (MAG) data to see if any field-aligned currents could be detected. The measured magnetic field components, $B_{x}, B_{y}$, and $\mathrm{B}_{\mathrm{z}}$, are shown in Figure $2 \mathrm{~d}$ using the co-rotating coordinate system described in Figure 1. The components labeled "model," are based on an offset magnetic dipole aligned along Saturn's rotational axis. The magnetic moment has been adjusted slightly to give agreement with the locally measured magnetic field. Note that the $\mathrm{B}_{\mathrm{z}}$ (model) field is negative, $-325 \mathrm{nT}$, indicating a southward field, as expected, since Saturn's magnetic dipole is directed northward. Also, note that the $\mathrm{B}_{\mathrm{y}}$ (model) field is positive, $+18 \mathrm{nT}$. This component arises from the $0.037 \mathrm{R}_{\mathrm{S}}$ northward offset of the magnetic dipole. As can be seen, there are small perturbations from the model field in the vicinity of the moon. These perturbations are of two types: smooth variations on spatial scales comparable to the distance to the moon, and more rapid ramp-like variations indicating a southward nearly field-aligned current from about 02:09:45 to 02:090:58 UT and a comparable northward current from about 02:09:58

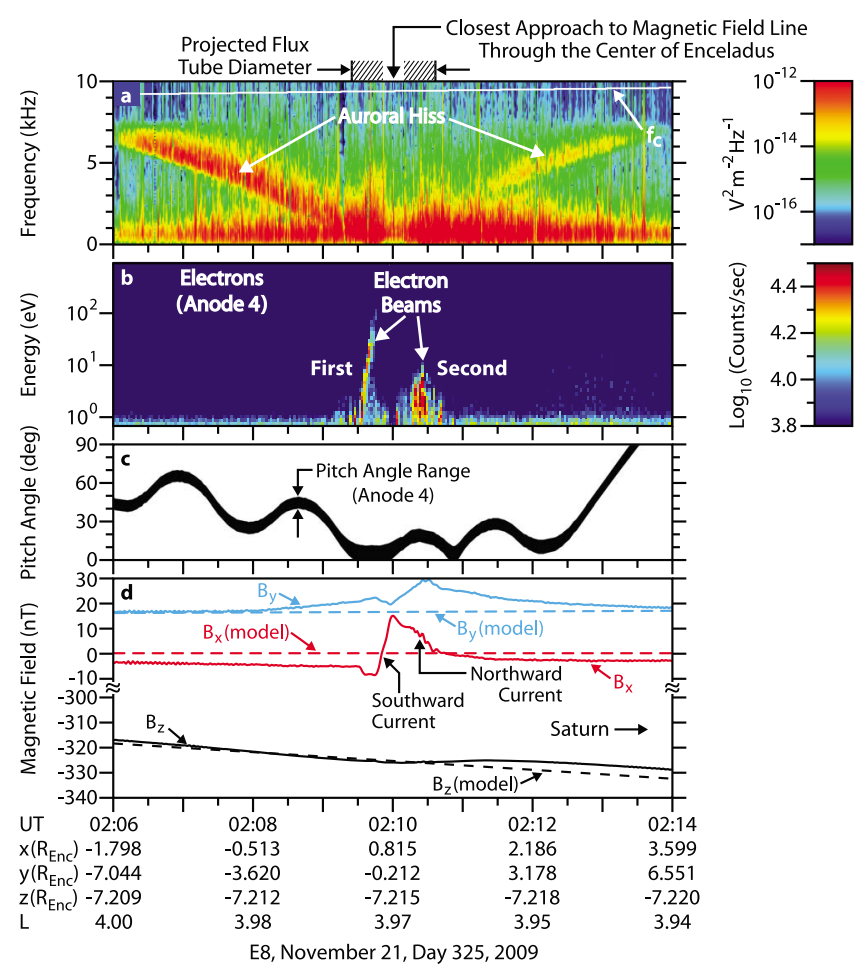

Figure 2. (a) An electric field frequency-time spectrogram from the RPWS instrument showing the V-shaped auroral hiss event observed during the E8 flyby; (b) an energy-time spectrogram from the CAPS electron spectrometer (ELS) showing two nearly field-aligned electron beams that are the likely source of the auroral hiss; (c) the pitch angle of the ELS observations; and (d) the magnetic field components, $B_{x}, B_{y}$, and $B_{z}$, measured by the Cassini magnetometer (MAG). The white line labeled $f_{c}$ in Figure $2 a$ is the electron cyclotron frequency. The magnetic field components labeled "model" in Figure 2d are for an offset dipole model of Saturn's magnetic field. 

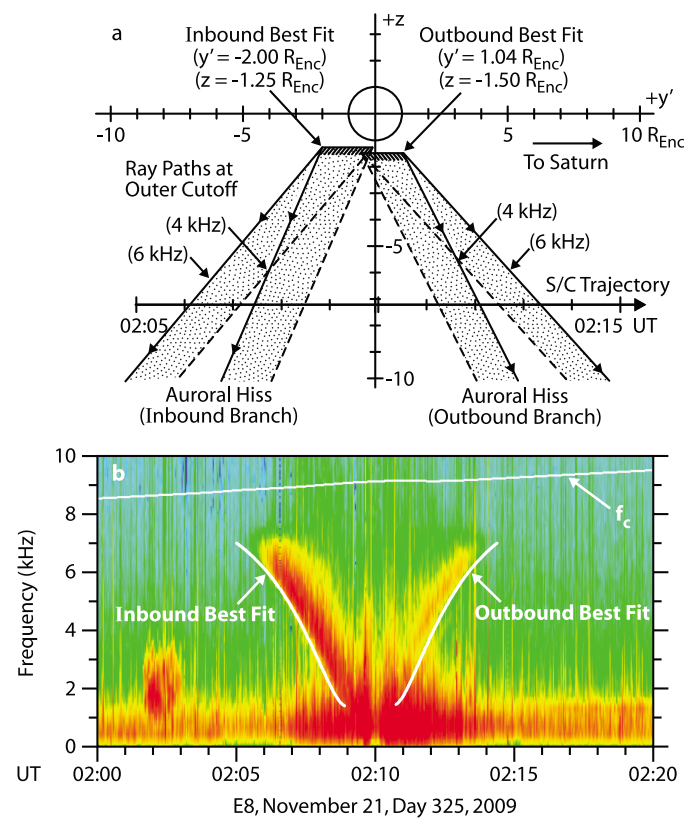

Figure 3. (a) The best-fit ray paths for the inbound and outbound sources; and (b) the corresponding fits (white lines) to the outer envelopes of the auroral hiss spectrum. The finite duration of the emission indicates that the sources extend over finite regions, as indicated by the hatched horizontal lines.

to $02: 10: 32$ UT. The slow variations are due to the deviation of the plasma flow around the moon [Dougherty et al., 2006], and the ramp-like variations are interpreted as nearly field-aligned currents associated with a standing shear-mode Alfvén wave excited by the moon, similar to that generated by Jupiter's moon Io [Goldreich and Lynden-Bell, 1969]. The northward current is on the Saturn-facing side of Enceladus, and the southward current is on the opposite side, as would be expected for an Alfvén wave excited by the moon [Neubauer, 1980]. For a further discussion of the Alfvén wave interpretation, see the auxiliary material. Note that the second, southward-directed, electron beam is consistent with the northward direction of the current. However, the relationship of the first electron beam to the Alfvén wave is not so clear, as this beam starts in a brief region of northward current on the anti-Saturn facing side of the moon, and then extends into a region where $\mathrm{B}_{\mathrm{x}}$ and $\mathrm{B}_{\mathrm{y}}$ are constant, indicating no current.

\section{Location of the Auroral Hiss Source}

[7] Because auroral hiss is known to be propagating along the whistler-mode resonance cone, it is possible to determine the source location by analyzing the characteristic V-shape of the spectrum. For the E8 flyby the electron plasma frequency is much greater than the electron cyclotron frequency (see the auxiliary material). Under these conditions it can be shown [Xin et al., 2006] that the angle of the ray path, $\psi$, relative to the magnetic field is given by the simple equation $\sin \psi=f \mid f_{c}$, where $f$ is the wave frequency and $f_{c}$ is the electron cyclotron frequency. From this equation it is easy to see that the radiation from a point source is beamed along a funnel-shaped surface, the opening angle $\psi$ of which increases with increasing frequency. It is this frequency dependence that gives the auroral hiss its characteristic V-shaped spectrum. Since the magnetic field strength, hence the angle $\psi$, varies along the ray path, the exact shape of the funnel-shaped surface must be determined by numerical integration. Once the shape of the funnel has been determined it is relatively easy to determine the times at which the spacecraft enters and leaves the funnel-shaped surface.

[8] To determine the source position, the procedure that we have used is to compute ray paths from an assumed point source for a variety of frequencies, and then adjust the location of the source to give the best fit to the entry and exit times defined by the sharp outer envelope of the V-shaped spectrum. The result of this best-fit procedure is shown in Figure 3. Since the source positions can be most accurately determined in a plane that contains the spacecraft trajectory, we have displayed the source positions and ray paths in the $\left(y^{\prime}, z\right)$ plane, where the $y^{\prime}$ axis is parallel to the spacecraft trajectory at closest approach (shown red in Figure 1). To fix the source position in the transverse direction, which is poorly determined, we have assumed that the source is in a plane that passes through the center of the moon (i.e., the $x^{\prime}=0$ plane in the rotated $\left(\mathrm{x}^{\prime}, \mathrm{y}^{\prime}\right)$ system, see Figure 1). To fit the envelope of the observed spectrum we found that two source positions were required, one that gives the best fit during the inbound pass, and the other that gives the best fit during the outbound pass. For the inbound pass the best fits are at $y^{\prime}=-2.0 R_{E n c}$ and $z=-1.25 R_{E n c}$, and for the outbound pass the best fits are at $y^{\prime}=1.04 R_{E n c}$ and $z=-1.5 R_{E n c}$. As can be seen in Figure $3 b$, the fits to the outer envelopes of the spectrum are very good. In the $y^{\prime}, z$ plane the uncertainty in the source position is about $\pm 0.5 \mathrm{R}_{\mathrm{Enc}}$, and in the transverse $\mathrm{x}^{\prime}$ direction the uncertainty is significantly larger, with $\mathrm{x}^{\prime}$ bounded by $-1.0 \leq \mathrm{x}^{\prime} \leq 2.0 \mathrm{R}_{\mathrm{Enc}}$. For a further discussion of the accuracy of this fitting procedure, including constraints on the transverse position, see the discussion in the auxiliary material. Since the emission extends for a substantial region inside of the white lines, it is clear that the source extends over a finite region in the $\mathrm{y}^{\prime}$ direction, as indicated by the two hatched horizontal lines, as well as southward along the magnetic field lines.

\section{Implications for Electron Acceleration}

[9] In order to generate whistler-mode emissions via a beam-plasma instability the reduced one-dimensional electron velocity distribution must have a region of positive slope. Although a plot of the electron counting rate as a function of velocity shows a well-defined peak, see Figure $2 b$, when the counting rate is converted to a velocity space distribution function there is no region of positive slope (see Figure S5 in the auxiliary material). This means that auroral hiss is not being generated near the spacecraft. That the electron velocity distribution function is stable at the spacecraft may seem surprising. However, it is entirely consistent with the observations, since the ray path analysis shows that the source is located very close to the moon. If the radiation were being generated all along the magnetic field line from the moon to the spacecraft, then the spectrum would be filled in uniformly between the outer envelopes of the emission, which it is not. Thus, we conclude that the region of positive slope, technically a "beam" in the plasma physics 
sense, only exists in a very limited region near the moon, probably at a distance of not more than 1 to $3 \mathrm{R}_{\mathrm{Enc}}$ above the surface. Beyond this region the positive slope is probably eliminated via wave-particle interactions, i.e., quasi-linear diffusion. Since a region of positive slope can only be maintained in steady state by a parallel electric field, we can then infer that the auroral hiss source defines the primary region where the electrons are being accelerated. Since the southward moving electrons are associated with the standing Alfvén wave, we believe that the electrons are most likely accelerated by parallel electric fields associated with the Alfvén wave. Both for Earth's aurora [Lysak and Song, 2003] and Jupiter's moon Io [Hill et al., 1983; Su et al., 2006] there are well developed theories showing that Alfvén waves can produce parallel electric fields via kinetic and electron inertial effects. However, we cannot rule out the possibility that the electron acceleration could be caused by some other mechanism, such as charging at the surface of the moon, or charging of small particles in the water vapor plume [Farrell et al., 2010].

[10] Acknowledgments. The research at the University of Iowa was supported by JPL contract 1415150 , and the research at the Southwest Research Center was supported by JPL contract 1405851 . The research at University College of London was supported by STFC and UKSA, and G. Jones acknowledges support via an STFC Advanced Fellowship.

[11] The Editor thanks William Farrell and Christopher Chaston for their assistance in evaluating this paper.

\section{References}

Dougherty, M. K., et al. (2006), Identification of a dynamic atmosphere at Enceladus with the Cassini magnetometer, Science, 311, 1406-1409, doi:10.1126/science.1120985.

Ergun, R. E., et al. (2003), Fast auroral snapshot satellite observations of very low frequency saucers, Phys. Plasmas, 10, 454-462, doi:10.1063/ 1.1530160 .

Farrell, W. M., W. S. Kurth, R. L. Tokar, J.-E. Wahlund, D. A. Gurnett, Z. Wang, R. J. MacDowall, M. W. Morooka, R. E. Johnson, and J. H. Waite Jr. (2010), Modification of the plasma in the near-vicinity of Enceladus by enveloping dust, Geophys. Res. Lett., 37, L20202, doi:10.1029/ 2010GL044768.

Goldreich, P., and D. Lynden-Bell (1969), Io, a Jovian unipolar inductor, Astrophys. J., 156, 59-78, doi:10.1086/149947.
Gurnett, D. A. (1966), A satellite study of VLF hiss, J. Geophys. Res., 71, 5599-5615.

Gurnett, D. A., and L. A. Frank (1972), VLF hiss and related plasma observations in the polar magnetosphere, J. Geophys. Res., 77, 172-190, doi:10.1029/JA077i001p00172.

Gurnett, D. A., S. D. Shawhan, and R. R. Shaw (1983), Auroral hiss, $\mathrm{Z}$ mode radiation, and auroral kilometric radiation in the polar magnetosphere: DE 1 observations, J. Geophys. Res., 88, 329-340, doi:10.1029/ JA088iA01p00329.

Hill, T. W., et al. (1983), Magnetosphere models, in Physics of the Jovian Magnetosphere, edited by A. J. Dessler, pp. 353-394, Cambridge Univ. Press, Cambridge, U. K., doi:10.1017/CBO9780511564574.012.

Lysak, R. L., and Y. Song (2003), Kinetic theory of Alfvén wave acceleration of auroral electrons, J. Geophys. Res., 108(A8), 1327, doi:10.1029/ 2003JA009859.

Maggs, J. E. (1976), Coherent generation of VLF hiss, J. Geophys. Res., 81, 1707-1724, doi:10.1029/JA081i010p01707.

Mosier, S. R., and D. A. Gurnett (1969), VLF measurements of the Poynting flux along the geomagnetic field with the Injun 5 satellite, J. Geophys. Res., 74, 5675-5687, doi:10.1029/JA074i024p05675.

Neubauer, F. M. (1980), Nonlinear standing Alfvén wave current system at Io: Theory, J. Geophys. Res., 85, 1171-1178, doi:10.1029/ JA085iA03p01171.

Porco, C. C., et al. (2006), Cassini observes the active south pole of Enceladus, Science, 311, 1393-1401, doi:10.1126/science.1123013.

Pryor, W. R., et al. (2011), The Enceladus auroral footprint at Saturn, Nature, in press.

Smith, B. A., et al. (1981), Encounter with Saturn: Imaging science results, Science, 212, 163-191, doi:10.1126/science.212.4491.163.

Su, Y.-J., S. T. Jones, R. E. Ergun, F. Bagenal, S. E. Parker, P. A. Delamere, and R. L. Lysak (2006), Io-Jupiter interaction: Alfvén wave propagation and ionospheric Alfvén resonator, J. Geophys. Res., 111, A06211, doi:10.1029/2005JA011252.

Tokar, R. L., et al. (2006), The interaction of the atmosphere of Enceladus with Saturn's plasma, Science, 311, 1409-1412, doi:10.1126/science. 1121061.

Xin, L., D. A. Gurnett, and M. G. Kivelson (2006), Whistler mode auroral hiss emissions observed near Jupiter's moon Io, J. Geophys. Res., 111, A04212, doi:10.1029/2005JA011411.

T. F. Averkamp, D. A. Gurnett, G. B. Hospodarsky, W. S. Kurth, J. S. Leisner, A. M. Persoon, and P. Schippers, Department of Physics and Astronomy, University of Iowa, Iowa City, IA 52242, USA. (donaldgurnett@uiowa.edu)

A. J. Coates and G. H. Jones, Mullard Space Science Laboratory, University College London, Holmbury St. Mary, Dorking RH5 6NT, UK. F. J. Crary, Southwest Research Institute, PO Drawer 28510, San Antonio, TX 78238, USA.

M. K. Dougherty, Blackett Laboratory, Imperial College London, London SW7 2AZ, UK. 